\title{
Estrés laboral, sensibilidad egocéntrica negativa y salud en profesionales venezolanos
}

\section{Job stress, negative egocentric sensitivity and healthy in Venezuelan professionals}

\author{
Lila Goncalves ${ }^{1}$, Lya Feldman y Leticia Guarino \\ Departamento de Ciencia y Tecnología del Comportamiento \\ Universidad Simón Bolívar, Venezuela
}

(Recepción: Enero 2009 - Aceptación: Mayo 2009)

\begin{abstract}
Resumen
El objetivo de la presente investigación fue determinar la relación existente entre el estrés laboral y la sensibilidad egocéntrica negativa con el estatus de salud física y psicológica de profesionales venezolanos. Se midieron el estrés laboral percibido, según el Modelo Demanda-Control (demanda-control-apoyo social), la sensibilidad egocéntrica negativa y el estatus salud física y psicológica autopercibida, en 441 profesionales venezolanos de diferentes disciplinas y de ambos sexos $(\mathrm{M}=220 ; \mathrm{F}=220$; edad $\mathrm{X}=30$ años, $\mathrm{DT}=6,52$ ). Los resultados mostraron que el estrés laboral y la sensibilidad egocéntrica negativa impactan sobre la salud autopercibida. La sensibilidad egocéntrica negativa se relaciona positivamente con mayores demandas laborales, así como con una mayor ansiedad, depresión, reportes de enfermedad, síntomas e indicadores de morbilidad y de manera inversa con control laboral, apoyo social, autoestima y bienestar, apoyando evidencias previas sobre el efecto pervasivo de la sensibilidad egocéntrica negativa sobre la salud y el bienestar de los individuos.
\end{abstract}

Palabras clave: estrés laboral, sensibilidad emocional, salud, profesionales.

\begin{abstract}
The aim of the present research was to determine the relationship among job stress and negative egocentric sensitivity, with the physical and psychological health status of Venezuelan professionals. The index of perceived job stress, using the Demand-Control Model (demand-control-social support), the negative egocentric sensitivity and the self perceived physical and psychological health status of Venezuelan professionals from different carriers and both genders were measured $(\mathrm{F}=220, \mathrm{M}=220$; mean age $=30, \mathrm{SD}=$ $6,52)$. Results showed that job stress and negative egocentric sensitivity had an important impact on health. Negative egocentric sensitivity was positively related to increased job demands, anxiety, depression, reports of illness, symptoms and indixes of morbidity, and inversely related to job control, job support, selfesteem and well-being, supporting previous findings about the pervasive effect of the negative egocentric sensitivity on health and well-being.
\end{abstract}

Key words: job stress, emotional sensitivity, health, professionals.

1 Correspondencia a: Lila Goncalves, Lya Feldman y Leticia Guarino. P.O. Box 89.000. Caracas 1080. Venezuela. Fax: (58 212) 9063594; 2864176. Teléfonos: (58 212) 9063593-95. E-mails: goncalvesl@usb.velfeldman@usb.ve lguarino@usb.ve 


\section{Introducción}

El estrés laboral es uno de los fenómenos más ampliamente estudiados en relación a las características del empleo, concebido en términos de un estado de tensión físico y mental que surge como consecuencia de un desequilibrio entre las demandas organizacionales y las capacidades del trabajador cuando éstas son sobre-utilizadas o infra-utilizadas. Más específicamente, Karasek y Theorell (1990) señalan que altas demandas laborales y poco control sobre su labor, son los elementos fundamentales para desencadenar elevados niveles de estrés en el trabajador, y esto a su vez tiene impacto negativo sobre la salud. Este modelo ha recibido suficiente respaldo empírico en múltiples investigaciones de salud ocupacional (Calnan, Wainwright, Forsthe, Wall \& Almond, 2001; Evans \& Steptoe, 2002; Karasek , Baker , Marxer , Ahlbom \& Theorell, 1998; Karasek \& Theorell, 1990; Mausner \& Eaton, 2000; Niedhammer \& Chea, 2003; Paterniti, Niedhammer, Lang, Consoli, 2002; Rydstedt,; Theorell et. al, 1998).

El Modelo de Demanda Control predice el estrés relacionado al riesgo de enfermedad, tanto física como psicológica, así como los activos y pasivos conductuales asociados al empleo (Levi, 2000). De esta forma, ha sido utilizado en estudios de estrés, fatiga, depresión, uso de medicamentos, ausencias laborales, accidentes en el lugar de trabajo, síntomas musco-esqueléticos, mortalidad, entre otros (Kristensen, 1995).

El Apoyo Social se incluyó posteriormente, como un tercer elemento en el modelo, introducido por Johnson y Hall (1988, c.p. Levi, 2000) como un factor protector, modificador del efecto de la alta tensión. Existe gran evidencia empírica que respalda tanto el efecto directo como el efecto moderador del apoyo social en su relación con el estrés y salud (Adler \& Mathews, 1994; Cohen \& Willis, 1985; Daniels \& Guppy, 1994; Dorman \& Zapf, 1999; Meneses, Feldman \& Chacon, 1999; Turner \& Marino, 1994).

También se han estudiado variables sociodemográficas asociadas al estrés laboral, como el género, nivel educativo, la edad, estado civil, entre otras, con el objeto de conocer cuáles de estas variables pueden ser consideradas como factores protectores o de riesgo para la salud del individuo. En este sentido, Begley y Czajka (1993) encontraron asociaciones directas entre el género y el nivel de estrés en sus investigaciones, por cuanto las mujeres se estresan más que los hombres. Lo mismo ocurre para el nivel de instrucción, donde a mayor nivel menor estrés, debido a que el individuo cuenta probablemente con más recursos para enfrentarlo. Maciejewski, Prigerson y Mazure (2001) encontraron que las mujeres de mayor edad con bajo nivel socioeconómico, que no estaban casadas, reportaban más estados depresivos y peores condiciones de salud que los hombres.

Por otro lado, dentro del modelo transaccional del estrés propuesto por Lazarus y colaboradores (ver Folkman \& Lazarus, 1985; Lazarus, 1981; Lazarus y Folkman, 1986; Lazarus, Kanner \& Folkman, 1980; Lazarus \& Launier, 1978; Steptoe, 1991), la relación entre los estímulos o demandas ambientales y sus consecuencias adaptativas puede estar moderada por diferencias individuales que afectan la evaluación cognitiva y el afrontamiento.

En el marco de este modelo, Guarino (2004) propuso un nuevo constructo de responsividad emocional denominado Sensibilidad Emocional, el cual redefine las implicaciones que una alta reactividad emocional tiene sobre la salud de los individuos sometidos a grandes demandas ambientales (ver Guarino, 2004; 2005). Dentro de este constructo, la dimensión más implicada en la relación estrés-enfermedad es la Sensibilidad Egocéntrica Negativa - SEN- actuando precisamente como moderadora de dicha relación, de tal manera que a mayor demanda ambiental y a mayor expresión de esta diferencia individual, mayor el deterioro físico y psicológico reportado por el individuo (Bethelmy y Guarino, 2008a,b; Guarino 2004; 2005; Herrera y Guarino, 2008).

Sobre la base de los anteriores hallazgos, este estudio se planteó la importancia de continuar la investigación sobre los factores de riesgo o protectores de la salud de los trabajadores, especialmente de profesionales universitarios venezolanos, por contar con pocas evidencias en nuestro contexto. De esta manera, se propuso determinar la relación del estrés laboral (Modelo Demanda-Control), 
la sensibilidad egocéntrica negativa y la salud, tanto física como psicológica, en una muestra de profesionales venezolanos de diferentes disciplinas. Los resultados obtenidos permitirán ampliar el conocimiento de la asociación entre las variables psicosociales y diferencias individuales vinculadas a la salud física y psicológica de los trabajadores, permitiendo con ello el desarrollo de programas preventivos y de intervención inmediata dirigidos a minimizar las consecuencias negativas e incrementar el estado de bienestar laboral.

\section{Método}

\section{Participantes}

La muestra estuvo constituida por 441 profesionales de ambos sexos, con edades comprendidas entre 22 y 60 años, con un promedio de edad de 30 años y una DT de 6,52. Se observó una distribución equitativa en cuanto al género, presentándose 220 hombres y 220 mujeres (49,9\% respectivamente). Para el momento de la investigación, los participantes se encontraban realizando estudios de postgrado en dos universidades venezolanas (Universidad Católica “Andrés Bello" $(\mathrm{N}=237)$; Universidad Simón Bolívar (N=204)), paralelamente a su desempeño laboral. Con respecto a las características laborales, el 42,4\% del grupo pertenece a ocupaciones que se ubican en la categoría de Ingeniería y Ciencias Aplicadas, 37\% a las Ciencias Sociales y Humanidades y 3,4 \% a carreras relacionadas a las Ciencias de la Salud. El número de meses en la ocupación estuvo mayormente distribuido en el período de 1-42 meses (41\%), seguido de un 37,2\% hasta 99 meses (8 años y 3 meses). Más de la mitad del grupo (55,8\%) se ubica dentro de un período de 1 a 42 meses de antigüedad en el trabajo actual, mientras un $27,7 \%$ dentro de 48 a 99 meses.

\section{Instrumentos}

Cuestionario de Estrés Laboral de Karasek y Theorell (1969). Este cuestionario en su versión original consta de 35 items acerca de la percepción personal del ambiente laboral, cantidad de trabajo realizado, satisfacción con el trabajo, apoyo del supervisor y compañeros, entre otras; en una escala tipo Likert de cuatro puntos que va de totalmente en desacuerdo a totalmente de acuerdo. A través de 12 preguntas adicionales evalúa indicadores de salud. Para fines de la presente investigación se empleó la versión abreviada del cuestionario de Karasek y Theorell (1990), la cual fue elaborada por Orth-Gomer y Wamala (1995) y ha sido utilizada en varias investigaciones (Feldman y cols, 1995; Feldman, Bagés y Vivas, 1997; Blanco, 2004; Gómez, Pérez, Feldman, Bagés y Vivas 2000). Consta de 17 items y tres subescalas: Demandas (items 1 al 5), decisión (6 al 11) y apoyo social (12 al 17). Se encontraron indices de consistencia interna adecuados (Alfa de Cronbach) que oscilan entre 0,72 y 0,87 , respectivamente.

Sub-escala de Sensibilidad Egocéntrica Negativa -SEN- (Guarino y Roger, 2005). La misma pertenece a la Escala de Sensibilidad Emocional ESE (para una descripción total de la escala ver Guarino y Roger, 2005). Específicamente la sub-escala SEN (22 items) describe la tendencia a reaccionar negativamente con emociones de aprehensión, rabia, desesperanza, vulnerabilidad y autocrítica ante los cambios ambientales y situaciones estresantes. Los autores reportaron un índice test-retest para esta sub-escala de $\mathrm{r}^{\mathrm{tt}}=0.718$; mientras que la consistencia interna se ubicó en $\alpha=$ 0,824 .

Autopercepción de salud física: se empleó la escala construida por Angelucci (2001) que mide la salud física percibida por el sujeto en función de dos aspectos relacionados con la salud en el último año: frecuencia en que alguna enfermedad interfiere con las actividades diarias del sujeto y frecuencia de la aparición de diferentes síntomas físicos o enfermedades. El coeficiente Alfa de Cronbach obtenido fue de 0.845 .

Salud Psicológica: para evaluar este aspecto se midieron las siguientes variabes: depresión, ansiedad, autoestima y bienestar. La depresión fue evaluada utilizando un cuestionario de síntomas depresivos tomado de Pearlin, Liberman, Menaghan y Mullan (1981). Este cuestionario consta de 10 ítems en su versión original, a ser respondidos en una escala de 4 puntos que va de 0 (No, nunca) 
a 3 (Mucho). Incluye preguntas sobre humor, problemas de sueño, apetito, energía, llanto, interés en actividades cotidianas y planes para el futuro. La adaptación al castellano en la muestra venezolana quedo reducida a 8 ítems obteniéndose un Alpha de Cronbach de 0,72 (Feldman, 2001). Ansiedad. Se utilizó la sub-escala de "estado" del Inventario de Ansiedad Estado-Rasgo (STAI -Spielberger, Gorsuch, Luchene, Vagg, Jacobs, 1983). La sub-escala aplicada consta de 20 ítems de 4 opciones de respuesta, que van de 1 (No, nunca) a 4 (Mucho). Estudios de confiabilidad y validez realizados con diferentes muestras y países de habla hispana han resultado satisfactorios según reportan DíazGuerrero y Spielberger (1975), Granell y Feldman (1981) y Feldman (2001). Autoestima. Se utilizó la escala de Rosenberg (1965) que aborda la autoestima como auto-aceptación. Esta escala consta de 10 ítems, cuyas opciones de respuesta van de 1 (extremadamente de acuerdo) a 4 (extremadamente en desacuerdo). En la adaptación al castellano en una muestra venezolana, tres ítems fueron eliminados por correlacionar muy bajo con el puntaje total, aunque su nivel de consistencia interna sigue siendo adecuado $(\alpha=0,85)$ (Feldman, 2001). Bienestar. El bienestar fue medido a través de un ítem ("Si usted tuviera que evaluar su nivel de bienestar en los últimos doce meses ¿Cómo lo calificaría en base a la siguiente escala?") a ser respondido en una escala de 10 puntos que va de 1 (Nada satisfactorio) a 10 (Muy Satisfactorio).

\section{Procedimiento}

Se realizaron contactos administrativos con los coordinadores del postgrado de ambas universidades. Posteriormente, se procedió a explicar los objetivos de la investigación, solicitando la participación voluntaria de los estudiantes y garantizando la confidencialidad de los resultados. Una vez obtenido el consentimiento informado, se procedió a la recolección de los datos mediante la administración de los instrumentos en forma grupal. Se hizo entrega del material a cada participante, con sus respectivas instrucciones, proporcionando un tiempo aproximado de 25 minutos para completar la información requerida.

\section{Resultados}

Los datos fueron analizados incialmente a través de estadísticos descriptivos para establecer su tendencia central y dispersión, para posteriormente analizar las asociaciones entre las variables a través del método de correlación $r$ de Pearson.

\section{Análisis Descriptivo}

Los resultados de la Tabla 1 sugieren que el grupo de participantes del estudio percibe tener unas demandas laborales relativamente altas, pero también un alto control o capacidad de decisión en las actividades que realiza en su trabajo, un nivel moderado de apoyo social, así como una baja sensibilidad egocéntrica negativa, si se comparan los promedios con el puntaje máximo posible. La dispersión de los datos es mayor en la sensibilidad egocéntrica negativa y en el control laboral.

Tabla 1 - Estadísticos Descriptivos de las Dimensiones del Cuestionario de Estrés Laboral y Sensibilidad Egocéntrica Negativa

\begin{tabular}{llllc}
\hline $\begin{array}{l}\text { Dimensiones } \\
\text { Estrés Laboral }\end{array}$ & Media & $\begin{array}{l}\text { Desviación } \\
\text { Estándar }\end{array}$ & $\begin{array}{l}\text { Rango } \\
\text { Observado }\end{array}$ & Rango Posible \\
\hline $\begin{array}{l}\text { Demandas Laborales } \\
\text { Control Laboral }\end{array}$ & 14,46 & 1,99 & $8-20$ & $1-20$ \\
$\begin{array}{l}\text { Apoyo Social } \\
\text { Sensibilidad Egocéntrica }\end{array}$ & $7,6,15$ & 3,53 & $14-35$ & $1-36$ \\
Negativa & 6,07 & 1,48 & $3-12$ & $1-12$ \\
\hline
\end{tabular}

$\mathrm{N}=441$ 
Con respecto a las variables de salud física, sesenta y cuatro por ciento del grupo ha consultado al médico de una a tres veces en el último año. Más de la mitad (53,5\%), presentó ausentismos laborales por problemas de salud con una frecuencia de una a tres veces en el último año, 85,5\% ha tenido que tomar medicamentos para el alivio de síntomas y 53,5\% ha sufrido de alguna enfermedad que le imposibilite la realización de sus actividades diarias de una a tres veces en el último año. No obstante, la mayoría del grupo (77,3\%) no ha tenido ninguna hospitalización (Ver Tabla 2).

Tabla 2 - Porcentajes relacionados a los Indicadores de Morbilidad

\begin{tabular}{ll}
\hline Frecuencia de consultas médicas & \\
Ninguna & $24,7 \%$ \\
1 - 3 veces & $63,7 \%$ \\
4 - 6 veces & $8,6 \%$ \\
7 ó más veces & $2,5 \%$ \\
\hline Frecuencia de hospitalizaciones & \\
Ninguna & $77,3 \%$ \\
1 - 3 veces & $21,1 \%$ \\
4 - 6 veces & $9 \%$ \\
7 ó más veces & \\
\hline Ausentismos laborales por problemas de salud & \\
Ninguna & $37,2 \%$ \\
1 - 3 veces & $53,5 \%$ \\
4 - 6 veces & $6,6 \%$ \\
7 ó más veces & $2,5 \%$ \\
\hline Tomar medicamentos para alivio de síntomas & \\
Ninguna & $13,8 \%$ \\
1 - 3 veces & $49,2 \%$ \\
4 - 6 veces & $16,3 \%$ \\
7 ó más veces & $20 \%$ \\
\hline Sufrir alguna enfermedad que imposibilite la realización de actividades diarias & \\
Ninguna & $39,9 \%$ \\
1 - 3 veces & $53,5 \%$ \\
4 - 6 veces & $4,5 \%$ \\
7 ó más veces & $1,8 \%$ \\
\hline
\end{tabular}

$\mathrm{N}=441$

En cuanto a los síntomas reportados, más de la mitad del grupo (76\%) padece de tensión muscular, el 63,9\% de dolor de cabeza, el 52,2\% de cólicos y ardor estomacal, el 50,8\% de olvidos y $48,1 \%$ de dificultades para concentrarse. Es relevante observar que los valores altos se aprecian especialmente en aquellos síntomas asociados a situaciones de tensión y estrés.

Por su parte, los descriptivos de las variables de salud psicológica indican que los participantes de este estudio reportan tener, en general, una buena salud psicológica. Los niveles de autoestima comprenden valores altos, mientras que los de ansiedad y depresión son moderados a bajos. De igual forma, la percepción del bienestar en general se ubica hacia el extremo superior (Tabla 3). 
Tabla 3 - Estadísticos descriptivos de Salud Psicológica

\begin{tabular}{lcccc}
\hline $\begin{array}{l}\text { Indicadores de } \\
\text { Salud Psicológica }\end{array}$ & Media & $\begin{array}{c}\text { Desviación } \\
\text { Estándar }\end{array}$ & $\begin{array}{c}\text { Rango } \\
\text { Observado }\end{array}$ & Rango Posible \\
Autoestima & 24,2471 & 3,1123 & $8-28$ & $1-28$ \\
Depresión & 13,5229 & 3,5689 & $8-26$ & $1-32$ \\
Ansiedad & 38,6962 & 9,8485 & $20-73$ & $1-80$ \\
Bienestar & 7,72 & 1,24 & $2-10$ & $1-10$ \\
\hline
\end{tabular}

Análisis de Correlaciones

Las correlaciones entre el conjunto de variables psicosociales y de salud, tanto física como psicológica, están representadas en la Tabla 4. Los datos indican que la sensibilidad egocéntrica negativa se relaciona de manera significativa e inversa con el apoyo social, con el control laboral , la autoestima y el bienestar. A su vez, una alta sensibilidad negativa se asocia con altas demandas laborales, mayor morbildad, mayor reporte de enfermedades y síntomas, así como con mayor ansiedad y depresión.

Por su parte, demandas laborales más altas se relacionan de manera significativa y positiva con el control laboral, con indicadores de morbilidad, con reportes de enfermedad y síntomas y con mayor ansiedad. El control laboral se asocia inversamente con el reporte de enfermedades, síntomas, ansiedad y depresión y de forma directa con la autoestima y el bienestar.

Finalmente, las personas con mayor apoyo social presentan menores reportes de enfermedades y síntomas, menores niveles de ansiedad y depresión, así como mayor autoestima y bienestar.

Tabla 5 - Matriz de correlación entre las variables del estudio

\begin{tabular}{|c|c|c|c|c|c|c|c|c|c|c|}
\hline & $\begin{array}{l}\text { Dem. } \\
\text { Lab. }\end{array}$ & $\begin{array}{l}\text { Cont. } \\
\text { Lab. } \\
\end{array}$ & $\begin{array}{l}\text { Apoyo } \\
\text { Social } \\
\end{array}$ & Morb. & Enferm. & Sínt. & Ans. & Dep. & Autoest. & Bienest. \\
\hline $\begin{array}{l}\text { Sensibilidad } \\
\text { Negativa }\end{array}$ & $.119 *$ & $-.171^{* *}$ & $-.214^{*}$ & $.239 * *$ & $.319 * *$ & $.410^{* *}$ & $.597 * *$ & $.578^{* *}$ & $-.391 * *$ & $-.439 * *$ \\
\hline $\begin{array}{l}\text { Demandas } \\
\text { Laborales }\end{array}$ & 1 & $.131^{* *}$ & -.028 & $.132 * *$ & $.230^{* *}$ & $.192 * *$ & $.207^{* *}$ & .092 & .027 & -.046 \\
\hline $\begin{array}{l}\text { Control } \\
\text { Laboral }\end{array}$ & & 1 & $.423 * *$ & -.098 & $-.106^{*}$ & $-.142 * *$ & $-.268 * *$ & $-.209 * *$ & $.230 * *$ & $.247 * *$ \\
\hline Apoyo Social & & & 1 & -.045 & $-.111 *$ & $-.145^{* *}$ & $-.280 * *$ & $-.185^{* *}$ & $.234^{* *}$ & $.215^{* *}$ \\
\hline Morbilidad & & & & 1 & $.491^{* *}$ & $.397 * *$ & $.225 * *$ & $.269 * *$ & -.069 & $-.202 * *$ \\
\hline Enfermedades & & & & & 1 & $.580 * *$ & $.366^{* *}$ & $.331^{* *}$ & $-.143^{* *}$ & $-.318^{* *}$ \\
\hline Síntomas & & & & & & 1 & $.440^{* *}$ & $.433 * *$ & $-.165^{* *}$ & $-.288 * *$ \\
\hline Ansiedad & & & & & & & 1 & $.631^{* *}$ & $-.413^{* *}$ & $-.623 * *$ \\
\hline Depresión & & & & & & & & 1 & $-.436 * *$ & $-.501 * *$ \\
\hline Autoestima & & & & & & & & & 1 & $.384^{* *}$ \\
\hline Bienestar & & & & & & & & & & 1 \\
\hline
\end{tabular}

Dem. Lab. = Demandas laborales; Cont. Lab.= Control laboral; Morb. = Morbilidad; Enferm. = Frecuencia de enfermedades; Síntomas = Frecuencia de síntomas; Ans. = Ansiedad; Dep. = depresión; Autoest. = Autoestima; Bienest. $=$ Bienestar

$* \mathrm{p}<.05, * * \mathrm{p}<.01$, 


\section{Discusión}

El objetivo de la presente investigación fue determinar la relación entre el estrés laboral y la sensibilidad egocéntrica negativa, como diferencia individual, con la salud física y psicológica autopercibida en una muestra de profesionales venezolanos.

Los resultados obtenidos confirman la asociación entre el estrés laboral y la salud, tanto física como psicológica, con base al modelo de Demanda-Control desarrollado por Karasek y Theorell (1990) y coinciden con otras investigaciones que destacan el efecto negativo de la sobrecarga laboral, el bajo nivel de control y autonomía sobre las condiciones laborales, así como el impacto del bajo apoyo social en el desarrollo de problemas de salud y enfermedades (Akerboom \& Maes, 2006; Eriksen, Ihlebaek, Jansen \& Burdorf, 2006; Escribá-Agüir \& Pérez-Hoyos, 2007; Evans \& Steptoe, 2002; Juárez-García, 2005; Karasek Baker, Marxer, Ahlbom \& Theorell, 1981; Niedhammer \& Chea, 2003; Rydstedt, Johansson \& Evans, 1998; Rydstedt, Devereux \& Sverke, 2007;).

En cuanto a las demandas laborales, éstas se asociaron de manera directa con todos los indicadores de salud física utilizados. al igual que con mayores síntomas tanto físicos como psicológicos, ansiedad y depresión. Estas asociaciones han sido corroboradas en otras investigaciones las cuales coinciden en afirmar que la sobrecarga laboral constituye una importante fuente de estrés debido a sus consecuencias sobre la salud y el bienestar (Feldman, 2001; Frankenhaeuser, 1991; Goncalves, 2004; Karasek \& Theorell, 1990; Rydstedt, Johansson \& Evans, 1998). Karasek et al. (1981) encontraron que un incremento en las demandas laborales combinado con baja capacidad de decisión está asociado a un mayor reporte de síntomas y aumenta el riesgo de desarrollar dolencias coronarios y prematura muerte cerebrovascular (Bergman, Ahmad y Stewart, 2003), al igual que genera transtornos del sueño (Akerstedt, Knutsson, Westerholm, Theorell, Alfredsson y Kecklund,2002).

En cuanto al control laboral, se asoció positivamente con la autoestima y el bienestar y de forma negativa con depresión, ansiedad, indicadores de morbilidad y reporte de síntomas. Muchas investigaciones destacan la importancia del control laboral como factor protector debido a su relación, no solo con la salud, sino con la productividad y satisfacción en el trabajo (Escribá-Agúir y Pérez-Hoyos, 2007; Feldman at al. 2005; Karasek \& Theorell, 1990; opus cit; Noor, 1995; Parkes, 1989). Poder tomar decisiones en el trabajo se ha encontrado asociado también a una alta autoestima, pero cuando ello envuelve fuertes responsabilidades, entonces también puede transformarse en una fuente de ansiedad (Calnan et al., 2001; Feldman, 2001). Otros autores reportaron el impacto de la alta capacidad de decisión y control como factor protector de la depresión, pero que a su vez, su ausencia o baja presencia contribuye al desarrollo de problemas de salud (Mausner y Eaton, 2000; Mursula, Vahtera, Pentti y Kivimäky, 2004).

En cuanto al apoyo social laboral, como tercera dimensión del Modelo de Karasek y Theorell (1990), los resultados obtenidos destacaron su importancia como factor protector de la salud. Un mayor apoyo social en el trabajo, se asoció con mayor autoestima y bienestar, a la vez que con menor ansiedad. Estos resultados apoyan los hallazgos obtenidos por Evans y Steptoe (2002), donde la presencia de apoyo social se asoció con menores niveles de ansiedad.

Dentro del ambiente laboral la falta de apoyo del supervisor ha demostrado tener un impacto importante sobre la salud mental y fisíca de sus supervisados (Van der Ploeg y Kleber, 2003), produciendo disturbios del sueño y cansancio (Akerstedt et al (2002), al igual que mayores niveles de presión arterial (Wager, Fieldman y Hussey , 2003). Por otra parte, los supervisores desempeñan un papel importante debido a que brindan un apoyo instrumental y de información sobre las responsabilidades específicas del trabajo, ayudando a reducir la sobrecarga laboral y promoviendo sentimientos de valor y autoestima (Feldman, 2001).

En síntesis, el modelo de Demanda Control empleado en este estudio ha resultado ser muy útil para la compresión del estrés laboral desde un enfoque que contempla los elementos del entorno de trabajo incluyendo el apoyo social laboral y los resultados obtenidos permiten fortalecer y validar este modelo. 
Con respecto a la Sensibilidad Egocéntrica Negativa (SEN), los resultados de este estudio son consistentes con lo esperado, en tanto la SEN constituye una forma de reactividad emocional nada funcional para el individuo que ejerce un efecto perjudicial sobre su salud y bienestar. En este sentido, la SEN no sólo se asocia a deterioro en la salud física y mental de los individuos, efecto que ya ha sido determinado con otros grupos etarios de condición socio-laboral diferente como estudiantes (Guarino, 2005), cadetes (Herrera y Guarino) y médicos especialistas (Bethelmy y Guarino, 2008a,b), sino que tiende a afectar a la percepción misma de las demandas ambientales, en este caso laborales (alta demanda y bajo control). Esto permite asumir que la SEN podría estar funcionando como un factor moderador de la evaluación o percepción de las demandas de los individuos, y aunque esto no fue objetivo de este trabajo, posiblemente estaría también afectando a las estrategias de afrontamiento que estas personas asumen frente a tales demandas, lo cual en conjunto apoya la hipótesis planteada por Guarino (2004; 2005) sobre el rol moderador de esta variable en la relación estrés-enfermedad y de la cual se están obteniendo evidencias cada vez más consistentes (ver Guarino, 2004).

En relación a la asociación negativa observada en este estudio con el apoyo social, ya un estudio anterior había determinado que los individuos con alta SEN tienden a reportar poco apoyo social percibido. Específicamente, Guarino y Sojo (2009) observaron una correlación negativa entre esta dimensión de la sensibilidad y el miedo a la revelación (MR), dimensión perteneciente al cuestionario de Apoyo Social validado por los mismos autores y que describe la dificultad de los individuos para hablar abiertamente de sus sentimientos y emociones y su tendencia a inhibir tales expresiones en las circunstancias en las cuales el apoyo de otras personas es necesario. Tal asociación puede interpretarse como la dificultad que tienen los individuos con emocionalidad negativa alta a buscar y recibir el apoyo de los otros y a expresar su malestar cuando experimentan situaciones estresantes, lo cual a su vez agrava su cuadro de deterioro físico y mental y dificulta su adaptación.

En conclusión, los resultados de este estudio con respecto a la relación entre la sensibilidad egocéntrica negativa como diferencia individual, el estrés laboral y el estatus de salud de profesionales venezolanos, apoya los hallazgos hasta ahora reportados sobre el impacto negativo que una característica como ésta tiene sobre el bienestar de los individuos. En función de estos resultados puede asumirse que la SEN actúa por la vía de incrementar la percepción de demanda del ambiente laboral y de menor control sobre el mismo por parte de los individuos, incrementando a su vez su reactividad fisiológica, lo cual origina la manifestación de síntomas observada. Cabe destacar que una mayor reactividad fisiológica en los individuos con alta SEN ya fue determinada experimentalmente (Guarino, 2004), por lo cual esta explicación sobre la ruta de acción de la SEN sobre el estatus de salud se hace bastante factible. Se requieren, sin embargo, nuevos estudios que establezcan de forma estadística a través de análisis de ruta este posible modelo de interacción de la sensibilidad emocional, lo cual daría fortaleza y validez a la concepción de esta variable como factor moderador de la relación estrés-salud.

En función de estos resultados, se sugiere la necesidad de desarrollar programas de intervención con miras a minimizar los desencadenantes negativos en la salud física y psicológica de los empleados que experimentan estrés laboral. Dichos programas, deberían estar orientados a cubrir no solo las condiciones más optimas del ambiente laboral, sino también facilitar el desarrollo de estrategias de afrontamiento eficaces para favorecer el bienestar personal, tanto dentro como fuera del trabajo. Según Cunningham, Woodward, Shannon, MacIntosh, Lendrum, Rosenbloom y Brown (2002) los empleados en posiciones activas con más control sobre los cambios laborales son más propensos a participar en el gran cantidad de actividades de rediseño y sienten que hacen su mayor contribución al cambio organizacional. Coincidimos con lo propuesto por Dollard, Winefield, Winefield, Jonge, (2000) y Mimura y Griffiths (2003) quienes señalan que la reducción en los niveles de estrés y el incremento en la productividad, podrían ser alcanzados, no necesariamente por decremento de las demandas en el trabajo, sino más bien por incremento de los niveles de control y apoyo social. 


\section{Referencias}

Adler, N. y Matthews, K. (1994). Health psychology: Why some people get sick and some stay well. Annual Review of Psychology, 45, 229-259.

Angelucci, L. (2001) Influencia de las variables sociodemográficas, el apoyo social y locus de control de salud sobre la salud en empleados universitarios. Manuscrito no publicado, Universidad Simón Bolívar, Venezuela

Akerboom, S. \& Maes, S. (2006). Beyond demand and control: The contribution of organizational risk factors in assessing the psychological well-being of health care employees. Work \& Stress; 20(1):21-36.

Akerstedt, T. Knutsson, A. Westerholm, T. Theorell, T. Alfredsson, L. y Kecklund, G. (2002). Sleeep disturbances, work stress and work hours: A cross-sectional study. Journal of Psychosomatic Research, 53: 741 - 748.

Begley, T.H. y Czajka, J.M. (1993). Panel análisis of the moderating effects of commitment on job satisfaction, intent to quit, and health following organizational change. Journal of Applied Psychology, 78, 4, 552-556.

Bergman, B. Ahmad, F y Stewart, D.E. (2003). Physician health, stress and gender at a university hospital. Journal of Psychosomatic Research, 54, 171-178.

Bethelmy, L. y Guarino, L. (2008). Estrés laboral, afrontamiento, sensibilidad emocional y síntomas físicos y psicológicos en médicos venezolanos. Revista Colombiana de Psicología. En prensa.

Calnan, M. Wainwright, D. Forsthe, M. Wall, B y Almond, S. (2001) Mental health and stress in the workplace: the case of general practice in the UK. Social Science \& Medicine, 52, 499-507.

Carrasquel, Y.C. (1997). Cualidad del rol, relación familia-trabajo, genero y salud en profesionales medicos. Trabajo no publicado presentado para optar al título de Magíster en Psicología.. Universidad Simón Bolívar. Caracas.

Cohen, S. y Willis, T. A. (1985). Stress, social support, and the buffering hypothesis. Psychological Bulletin, 98, 310-357.

Daniels, K. y Guppy, A. (1994). Ocupational stress, social support, job control, and pshychological wellbeing. Human Relations, 47, 1523-1541.

Degoratis, L., Lipman, R., Convi, L. y Rickles, K. (1971). Neurotic symptom dimensions. Archives of General Psychiatric, 24, 454-464.

Díaz-Guerrero y Spielberger (1975). IDARE. Inventario de ansiedad: Rasgo-Estado. Manual e Instructivo. México: El Manual Moderno, S.A.

Dorman, C. y Zapf, D. (1999). Social support, social stressors at work and depresive symptoms: Testing for main and moderating effects with structural equations in a three-wave longitudinal study. Journal of Aplied Psychology, 84, 874-884.

Eriksen, H.R.; Ihlebaek, C.; Jansen, J.P. \& Burdorf, A.(2006). The relations between psychosocial factors at work and health status among workers in home care organizations. International Journal of Behavioral Medicine, Vol. 13, № 3, 183-192.

Escribá-Agüir, V. y Pérez-Hoyos, S.(2007). Psychological well-being and psychological work environment characteristics among emergency medical and nursing staff. Stress and Health. 23: 153-160

Evans, O. y Steptoe, A. (2002) The contribution of gender - role orientation, work factor and hom stressors to psychological web-being and sickness absence in male and female dominated occupational groups. Social Science \& Medicine, 54, 481-492. 
Feldman, L. (2001). Social roles, psychosocial factors and health in Venezuelan working women. Tesis doctoral no publicada, Universidad de Southampton, Reino Unido.

Feldman, L.; Bagés, N. y Vivas, E. (1997). Riesgo de Salud en Mujeres con Roles Múltiples: Un Estudio Transcultural. Informe interno no publicado, Universidad Simón Bolívar, Sartenejas, Venezuela.

Feldman, L., Chacón, G., Blanco, G., Carrasquel, Y., y García, L. (1995). Proyecto Mujer Trabajo y Salud. Informe interno no publicado.Universidad Simón Bolívar, Sartenejas, Venezuela.

Folkman, S., y Lazarus, R. (1985). If it changes it must be a process: Study of emotion and coping during three stages of a college examination. Journal of Personality and Social Psychology, 48, 150-170.

Frankenhaeuser, M. (1991). The Psychophysiology of sex differences as related to occupational status. In U. Frankenhaeuser, U. Lundberg \& M. Chesney (Eds.), Women, Work and Health: Stress and Opportunities. New York: Plenum Press.

Goncalves, L. (2004). Estrés Laboral, Rabia, Apoyo Social y Salud. Tesis de Maestría no publicada. Universidad Simón Bolívar. Venezuela

Granell, E. y Feldman, L. (1981). Un inventario de temores para estudiantes niversitarios: Un estudio normativo. Psicología. 8 (2 y 3): 155-172.

Guarino, L. (2004). Emocional Sensitivity: a new measure of emotional lability and its moderating role in the stress-illness relationship. Tesis Doctoral Tesis doctoral sin publicar, Universidad de York, Inglaterra.

Guarino, L. (2005). Sensibilidad Emocional: Su Rol Moderador en la Relación Estrés-Enfermedad.

Psicología Conductual, 13 (2), 217-230.

Guarino, L. y Roger, D. (2005). Construcción y Validación de la Escala de Sensibilidad Emocional (ESE). Un nuevo enfoque para medir neuroticismo. Psicothema, 17 (3), 465-470.

Guarino, L., y Sojo, V. (2008). Adaptación y validación del ITQ (Interpersonal Trust Questionnaire). Una nueva medida del apoyo social. En preparación.

Herrera, V. y Guarino, L. (2008). Personalidad, estrés y salud en cadetes navales venezolanos. Universitas Psicológica, 17, 1, 185-198.

Juárez-García, A. (2005). Factores Psicosociales relacionados con la salud mental en profesionales de servicios humanos en México. Ciencia \& Trabajo, (6) 14 189-196.

Karasek, R.A. y Theorell, T. (1990). Healthy work: Stress, productivity, and the reconstruction of working life. New York: BasicBooks.

Karasek, R.A. (1998). Demand/Control Model: A social, emotional, and physiological approach to stress risk and active behaviour development. In J. M. Stellman (ed.), Encyclopaedia of occupational health and safety. Geneva: International Labour Office.

Karasek, R.A., Baker, D., Marxer, F., Ahlbom, A. y Theorell, T. (1998). Job decision latitude, job demands, and cardiovascular disease: a prospective study of Swedish men. American Journal of Public Health, 71, 7, 694-705.

Kristensen, T. (1995). The Demand-Control-Support model: Methodological challeges for future research. Stress Medicine , 11, 7-26.

Lazarus, R. (1981). The stress and coping paradigm. En C. Eisdorfer, D. Cohen, A. Kleinman, y P. Maxim (Eds.). Models for clinical psychopathology (pp. 177-214). New York: Sprectrum.

Lazarus, R., Kanner, A., y Folkman, S. (1980). Emotions: A cognitive-phenomenological analysis. En R. Plutchik y H. Kellerman (Eds.). Theories of emotion, Vol. 1: Emotion: Theory, research and experience (pp. 189-217). New York: Academic Press. 
Lazarus, R., y Folkman, S. (1986). Estrés y Procesos Cognitivos. Barcelona: Martinez Roca.

Lazarus, R., y Launier, R. (1978). Stress-related transactions between person and environment. En L. A. Pervin y M. Lewis (Eds.). Perspectives in interactional psychology (pp. 287-327). New York: Plenum.

Levi, L. (2000). Stressors at the workplace:Theoretical models. En P.Shanall, K. BelKie, P. Landbergis, D. Baker (Eds) Occupational Medicine. The Workplace and cardiovascular Disease: State of the Art Reviews. Philadelphia, Hanley \& Belfus, Inc.

Maciejewski, P., Prigerson, H. y Mazure, C. (2001). Sex differences in event-related risk for major depression. Psychological Medicine, 31, 593-604.

Mausner, H y Eaton, W. (2000). Association psychosocial work environment and depression: epidemiologic assessment of the demand-control model. American Journal of Public Health, 90 (11) 1765-1770.

Mursula, L. A., Vahtera, J., Pentti, J. y Kivimäki, M. (2004). Effect of employee worktime control on health: a prospective cohort study. Occupational and Environmental Medicine, 61, 254-261.

Niedhammer, I. y Chea, M. (2003). Psychosocial factors at work and self reported health: comparative results of cross sectional and prospective analyses of the French GAZEL cohort. Occupational and Environmental Medicine, 60, 509-515.

Noor, N.M. (1995). Job-role quality and women's psychological well-being: locus of control and social support as moderators. Journal of Community \& Applied Social Psychology, 5, 259-272

Orth-Gomer, K. y Wamala, S. (1995). Women's Work and health in various cultures. Proposed questionnaire. Internal report. Karolinska Institute. Suecia.

Parkes, K. (1989). Personal control in an occupational context. In A. Steptoe \& A. Appels (Eds.). Stress, Personal Control and Health. New York: John Wiley \& Sons.

Paterniti, S., Niedhammer, I., Lang, T., Consoli, S.(2002). Psychosocial factors at work, personality traits and depressive symptoms. The British Journal of Psychiatry, 181, 111-117.

Pearlin, L.I., Liberman, M.A., Menaghan, E.G. \& Mulan, J.T. (1981). The stress process. Journal of Health and Social Behavior, 22, 337-356.

Rosenberg, M. (1965). Society and the Adolescent Self-image. Princeton, NJ: Princeton University Press

Rydstedt, L.W., Johansson, G., y Evans, G.W. (1998). A longitudinal study of workload, health and well-being among male and female urban bus drivers. Journal of Occupational and Organizational Psychology, 71, 35 - 45.

Rydstedt, L.W.; Devereux, J. \& Sverke, M. (2007). Comparing and combining the demand-controlsupport model and the efford reward imbalance model to predict long-term mental strain. Europen Journal of Work and Organizational Psychology, 16 (3), 261-278

Spielberger, C.D., Gorsuch, R.L., Luchene, R.E. et al. (1983). Manual for the State-Trait Anxiety Inventory (revised edition). Palo Alto, CA: Consulting Psychologists Press.

Steptoe, A., (1991). The links between stress and illness. Journal of Psychosomatic Research, 35, 6, 633-644.

Theorell, T., Tsutsumi, A., Hallquist, J., Reuterwall, C., Hogstedt, C., Fredlund, P., Emlund, N., Jonson, J. (1998). Decision latitude, job strain, and myocardial infarction: A study of working men in Stockholm. American Journal of Public Health, 88, 3, 382-388.

Turner, R. y Marino, F. (1994). Social support and social structure: A descriptive epidemiology. Journal of Health and Social Behavior, 35, 193-212 
Van der Ploeg, E. y Kleber, R. J. (2003). Acute and chronic job stressors among ambulance personnel: predictors of health symptoms. Occupational and Environmental Medicine. [En línea]. Disponible en: http://oem.bmjjournal.com/cgi/content/full/60/suppl_1/i40

Wager, N. G., Fieldman and Hussey, T. (2003). The effect on ambulatory blood pressure of working under favourably and unfavourably perceived supervisors. Occupational and Environmental Medicine, 60, 468-474 